\section{Terahertz emission based on large-area photoconductive emitters illuminated via beam interference}

\section{N. Krauß ${ }^{\bowtie}$, M. Haas, S. Winnerl, M. Helm and T. Dekorsy}

\begin{abstract}
The use of beam interference in combination with a large-area photoconductive emitter for the generation of pulsed terahertz $(\mathrm{THz})$ radiation is presented. An interference pattern with a period twice that of the $\mathrm{THz}$ emitter is generated with a transmission phase grating, placed directly in front of the photoconductive emitter. This way, efficient THz generation is achieved with a single metallisation layer and a single lithography step in the fabrication technology of the $\mathrm{THz}$ emitter.
\end{abstract}

Introduction: The efficient generation and detection of broadband tera hertz $(\mathrm{THz})$ radiation has evolved as the driving force for future high speed $\mathrm{THz}$ time domain systems. Different generation mechanisms based on ultra short optical pulses such as optical rectification and photoconductive antennas have been investigated intensively during the past. Amplified high energy pulses with kilohertz pulse repetition rates are able to create ultra broadband $\mathrm{THz}$ radiation by optical rectifi cation in nonlinear crystals [1]. However, for many applications a higher data acquisition speed is favoured, thus higher pulse repetition frequen cies $(>\mathrm{MHz})$ should be used. These setups are mostly equipped with photoconductive emitters, which rely on linear infrared (IR) absorption and offer a high IR to $\mathrm{THz}$ conversion efficiency at moderate pulse energies. The emitted $\mathrm{THz}$ radiation in a single antenna is limited by the maximum thermal load and the breakdown voltage of the antenna. Power scaling was demonstrated via large area photoconductive emitters (LPEs) based on a periodic interdigitated metal semiconductor metal structure [2]. To prevent destructive inter ference of $\mathrm{THz}$ radiation in the far field from regions with inversed bias fields, every second electrode spacing needs to be covered with an additional electrically isolated opaque material. This way, uni directional charge carrier acceleration over the illuminated area is ensured. Unfortunately, the additional metallisation reduces the amount of absorbed light by a factor of 2 . Several variants of this con ventional LPE targeting higher conversion efficiencies were demon strated. Instead of an additional cover layer, the substrate in every second region can be removed $[3,4]$. This way, lower dark currents result, but a higher conversion efficiency is not to be expected. By implementing a micro lens array in front of the emitter structure, the amount of absorbed light is increased by a factor of 3 [5]. Further large area emitters rely on lateral photocurrents via the lateral photo Dember effect or Schottky fields at multiple asymmetric metal semiconductor junctions [6,7]. A fundamentally different approach relies on locally enhanced fields and absorption due to plasmonic effects [ 810$]$. Despite being very promising in terms of efficiency, plas monic structures need to be simulated and grown with high accuracy and are sensitive to the polarisation of the incident IR light.

The approach presented here is based on a biased LPE that is illu minated by a tailored interference pattern. This concept allows for an improved IR to THz conversion efficiency, while making an additional covering of every second electrode spacing unnecessary.

Illuminating photoconductive emitters via beam modulation: Interference produces a spatial intensity modulation that can be matched to the geo metry of the photoconductive emitter. In the case of a LPE, the interfer ence period along the direction (anti )parallel to the bias field must equal two times the period of the emitter. This situation is drawn schematically in Fig. 1 $a$. The intensity maxima of the interference pattern are placed at regions with equal bias field direction, whereas the minima of the inter ference pattern are placed at regions with the corresponding inverse bias field direction. Integrating the intensity over the absorbing regions reveals a total increase in absorption by a factor of about 2 compared with a con ventional LPE. In addition to that, the intensity within the relevant absorbing regions is increased by a factor of 2 compared with a Gaussian. A small fraction of $2.5 \%$ of the total incident power illuminates the electrode spacings with the reversed bias field direction, which will slightly reduce the emitted $\mathrm{THz}$ far field due to destructive interference.

Various techniques are available to produce interference patterns as shown in Fig. 1a. A simple, yet efficient and mechanically stable approach is to use a transmission phase grating in front of the LPE.
The working principle of a transmission phase grating is depicted in Fig. $1 b$. If the grating is illuminated by a single beam, the two first order diffracted beams will interfere directly after the grating. The theoretical maximum transmission efficiency into the two first orders is about $80 \%$, limited by diffraction into higher orders. Thus, taking all the discussed effects into account, an overall improvement in $\mathrm{THz}$ intensity of a factor of 2.7 compared with the conventional LPE [2] can be expected under equivalent experimental conditions, as long as no saturation occurs.
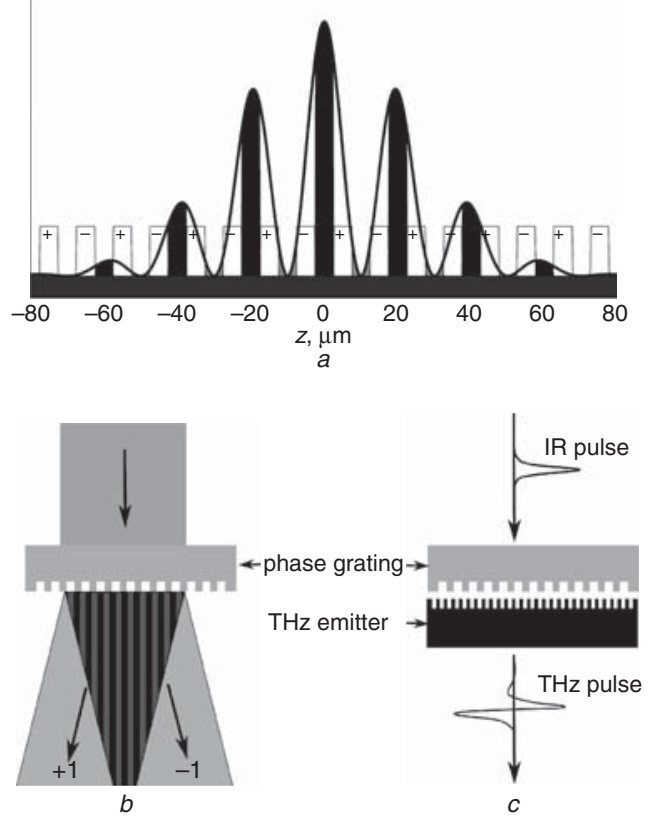

Fig. 1 Principle of illumination of large-area $\mathrm{THz}$ emitter by interference pattern (side-view) (Fig. la) (coloured areas indicate absorbed amount of light in two regions with different field directions (black and grey)); firstorder beam diffraction by phase grating and resulting interference pattern (Fig. 1b); schematic of implemented experimental configuration (Fig. 1c)

Experiment: The schematic setup for generating THz radiation is shown in Fig. 1c. To generate the interference pattern, a custom made trans mission phase grating, optimised for diffraction into the first orders at a central wavelength of $800 \mathrm{~nm}$ (Laser Laboratorium Göttingen e.V.), is implemented. The measured diffraction efficiency into the two first orders is about $75 \%$. The grating period is $40 \mu \mathrm{m}$, yielding an interfer ence period of $20 \mu \mathrm{m}$. The LPE consists of $5 \mu \mathrm{m}$ wide Au finger elec trodes, grown on an SI GaAs substrate with a period of $10 \mu \mathrm{m}$, compare Fig. 1a. For optimum alignment, the phase grating and the LPE are mounted on a rotation mount and a motorised linear translation stage, respectively. The incident pump beam is collimated to a spot size (full width at half maximum) of about $80 \mu \mathrm{m}$ onto the LPE with a pump power of about $600 \mathrm{~mW}$, corresponding to a pulse energy of $0.6 \mathrm{~nJ}$. The bias voltage on the LPE is adjusted to $9 \mathrm{~V}$. Field resolved detection of the $\mathrm{THz}$ pulses is done in a $1 \mathrm{~mm}$ thick $\mathrm{ZnTe}$ crystal. The experimental pump probe configuration is based on high speed asynchronous optical sampling, as described in $[11,12]$.

Results and discussion: Fig. 2 shows a typical THz transient, obtained at a data acquisition time of about $200 \mathrm{~s}$. Dry air is used to get rid of residual absorption due to water vapour in the air. The main $\mathrm{THz}$ pulse is followed by an echo, caused by internal reflection in the sub strate of the LPE. The power spectrum shows a dynamic range of more than $60 \mathrm{~dB}$ within a frequency range from 1 to $2 \mathrm{THz}$. Some remaining absorption lines due to water vapour are visible at about $3 \mathrm{THz}$. The highest resolvable frequency components are about $4 \mathrm{THz}$. A direct comparison with the transients obtained with a conven tional LPE under equivalent experimental conditions reveals transients and spectra with comparable amplitudes. The maximum theoretical improvement factor of about 2.7 is not reached. The THz intensity was limited in our case due to mechanical constraints that lead to a remaining distance between phase grating and the interdigitated struc ture. With increasing distance the $+/$ first order diffracted beams spatially separate after the grating such that the fraction of the beam that contributes to the interference pattern is decreased. 


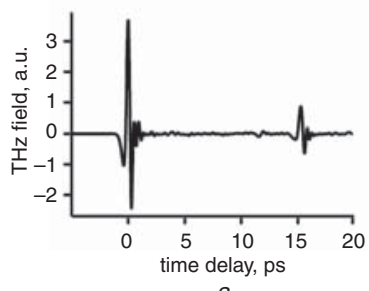

a

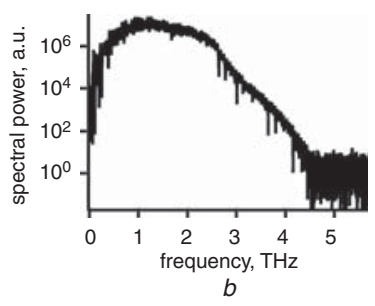

$b$
Fig. 2 Exemplary THz transient measured under nitrogen purging (Fig. 2a); corresponding spectral power at data acquisition time of $200 \mathrm{~s}$ (Fig. 2b)
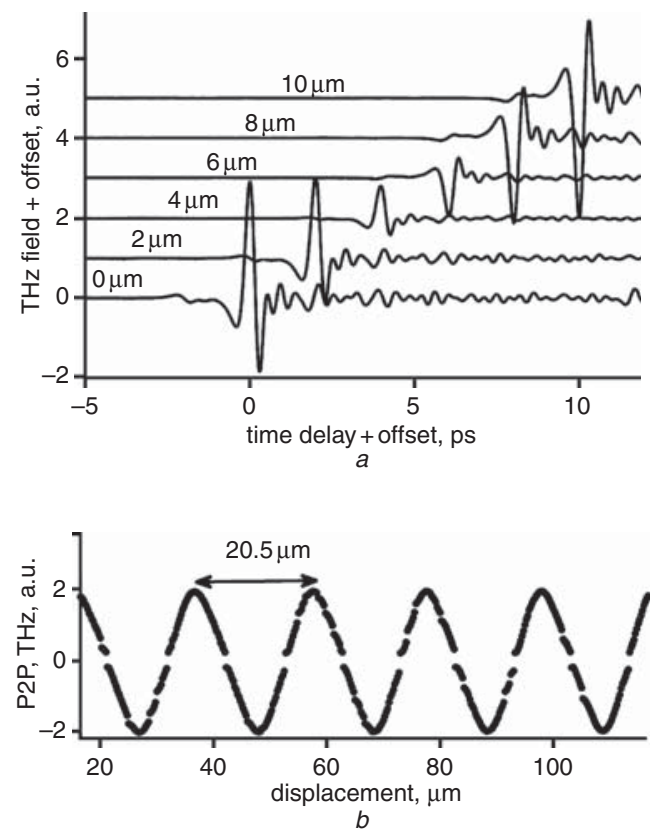

Fig. $3 \mathrm{THz}$ transients for different displacements between LPE and transmission grating, ranging from 0 to $10 \mu \mathrm{m}$ (Fig. 3a) (measurement performed in ambient atmosphere; offset added to both axes for clarity); peak-to-peak amplitude of $\mathrm{THz}$ transients against displacement (Fig. 3b)

In a second measurement we provided proof that the interference pattern illuminates every second active region of the LPE. To do so, the LPE is moved along the phase mask, orthogonal to the interference lines. From Fig. $1 a$ it can be seen that a displacement of half the LPE period should lead to a full cancellation of the $\mathrm{THz}$ in the far field due to a symmetric illumination of regions with different bias field direc tions. Doubling the displacement then swaps the interference maxima in field regions with the inversed electric field. This leads to a change in $\mathrm{THz}$ polarisation by $180^{\circ}$, which will manifest in the $\mathrm{THz}$ transients in the form of a sign flip.

The measurement result on such a spatial scan is shown in Fig. 3. The data clearly indicates an inversion of the THz polarisation, if a displace ment of about $10 \mu \mathrm{m}$ is applied. Fitting the THz amplitude against dis placement to a sinusoidal yields a period of $20.5 \mu \mathrm{m}$, which is in good agreement with the expected period of the emitter. Slight deviations from a sinusoidal result from the accuracy in displacement, which is limited by the translation stage to about $200 \mathrm{~nm}$. It can be observed that a complete cancellation of the $\mathrm{THz}$ field is not reached in the given measurement. This could be due to beam diffraction from the grating into the zero order or inhomogeneities of the emitter as well as the phase grating. This argument is supported by measurements on the LPE without the phase grating, which showed a non vanishing $\mathrm{THz}$ amplitude. In contrast to the $\mathrm{THz}$ field, the modulation of the photocurrent in dependency of the displacement is $<10 \%$, in agreement with theoretical calculations. It should be mentioned that the $\mathrm{THz}$ emitter exhibits sub $\mathrm{mA}$ dark currents throughout the measurements. This is in strong contrast to conventional LPEs, where the additional metallisation is not perfectly isolated, leading to typical leakage currents above $10 \mathrm{~mA}$. From this point of view, a much longer life time is expected from our device.

Conclusion: We have demonstrated the use of spatial beam intensity modulation by interference to illuminate a large area photoconductive emitter for efficient $\mathrm{THz}$ generation. This approach simplifies the fabri cation of the emitter, at the same time enabling an increased $\mathrm{THz}$ emis sion efficiency. The same principle can be applied in future experiments to increase the efficiency of large area photo Dember emitters, where no external bias is necessary, driving further applications of $\mathrm{THz}$ time domain spectroscopy and imaging systems.

Acknowledgments: This work was partially supported by the Center for Applied Photonics at the University of Konstanz. The authors thank L. Niemeyer for fruitful discussion.

N. Krauß, M. Haas and T. Dekorsy (Physics Department, University of Konstanz, Universitaetsstrasse 10, Konstanz 78457, Germany)

凶 E mail: nico.krauss@uni konstanz.de

S. Winnerl and M. Helm (Helmholtz Zentrum Dresden Rossendorf, Bautzner Landstrasse 400, Dresden 01328, Germany)

\section{References}

1 Löffler, T., Kreß, M., Thomson, M., Hahn, T., Hasegawa, N., and Roskos, H.G.: 'Comparative performance of terahertz emitters in amplifier-laser-based systems', Semicond. Sci. Technol., 2005, 20, p. 134

2 Dreyhaupt, A., Winnerl, S., Dekorsy, T., and Helm, M.: 'High-intensity terahertz radiation from a microstructured large-area photoconductor', Appl. Phys. Lett., 2005, 86, p. 121114

3 Awad, M., Nagel, M., Kurz, H., Herfort, J., and Ploog, K. 'Characterization of low temperature GaAs antenna array terahertz emitters', Appl. Phys. Lett., 2007, 91, p. 181124

4 Acuna, G.P., Buersgens, F.F., Lang, C., Handloser, M., Guggenmos, A., and Kersting, R.: 'Interdigitated terahertz emitters', Electron. Lett., 2008, 44, pp. 229231

5 Matthäus, G., Nolte, S., Hohmuth, R., Voitsch, M., Richter, W., Pradarutti, B., Riehemann, S., Notni, G., and Tünnermann, A.: 'Large-area microlens emitters for powerful $\mathrm{THz}$ emission', Appl. Phys. B, 2009, 96, pp. 233235

6 Klatt, G., Hilser, F., Qiao, W., Beck, M., Gebs, R., Bartels, A., Huska, K., Lemmer, U., Bastian, G., Johnston, M.B., Fischer, M., Faist, J., and Dekorsy, T.: 'Terahertz emission from lateral photo-Dember currents', Opt. Express, 2010, 18, pp. 49394947

7 Nagel, M.: 'German patent application'. DE 102012010926 A1

8 Berry, C.W., Wang, N., Hashemi, M.R., Unlu, M., and Jarrahi, M.: 'Significant performance enhancement in photoconductive terahertz optoelectronics by incorporating plasmonic contact electrodes', Nat. Commun., 2013, 4, p. 1622

9 Park, S.G., Jin, K.H., Yi, M., Ye, J.C., Ahn, J., and Jeong, K.H.: 'Enhancement of terahertz pulse emission by optical nanoantenna', ACS Nano, 2012, 6, pp. 20262031

10 Yardimci, N.T., Yang, S.H., Berry, C.W., and Jarrahi, M.: 'High-power terahertz generation using large-area plasmonic photoconductive emitters', IEEE Trans. Terahertz Sci. Technol., 2015, 5, pp. 223229

11 Bartels, A., Hudert, F., Janke, C., Dekorsy, T., and Köhler, K. 'Femtosecond time-resolved optical pump-probe spectroscopy at kilohertz-scan-rates over nanosecond-time-delays without mechanical delay line', Appl. Phys. Lett., 2006, 88, p. 04117

12 Klatt, G., Gebs, R., Janke, C., Dekorsy, T., and Bartels, A.: 'Rapid-scanning terahertz precision spectrometer with more than 6 THz spectral coverage', Opt. Express, 2009, 17, pp. 2284722854 